\title{
Automatic Color Extraction Algorithm of Graphic Design Image Based on Artificial Intelligence
}

\author{
Qian Zhao*, Hong Zhang \\ Jiujiang University, Jiujiang 332005, China \\ *Corresponding author: 51987313@qq.com
}

Received: August 7, 2021. Revised: December 27, 2021. Accepted: January 11, 2022. Published: January 12, 2022.

\begin{abstract}
The extraction of color features plays an important role in image recognition and image retrieval. In the past, feature extraction mainly depends on manual or supervised learning, which limits the automation of the whole recognition or retrieval process. In order to solve the above problems, an automatic color extraction algorithm based on artificial intelligence is proposed. According to the characteristics of BMP image, the paper makes use of the conversion between image color space and realizes it in the visual $\mathrm{C}++6.0$ environment. The experimental results show that the algorithm realizes the basic operation of image preprocessing, and realizes the automatic extraction of image color features by proper data clustering algorithm.
\end{abstract}

Keywords-Artificial Intelligence; Graphic Artist Designer; Color extraction.

\section{INTRODUCTION}

$\mathrm{T}_{\mathrm{i}}^{\mathrm{H}}$ HE characteristics of graphic design image mainly include color, form and texture. For color images, color is the most important and intuitive image feature. Color feature extraction plays an important role in image processing, recognition and retrieval. In the past, feature extraction was mainly realized by manual or supervised learning, which limited the automation of the whole recognition or retrieval process.

Color is one of the important factors to describe the emotion of a picture and painting. Different colors produce different emotional effects. Art and design often use color psychology to affect people's sensory experience. At present, there are two types of color extraction methods: color quantization algorithm and clustering algorithm. Color quantization algorithm mainly includes octree algorithm and median segmentation method, Clustering algorithm, taking clustering algorithm as an example, feature extraction is one of the key problems of pattern recognition [1], and data clustering does not need the information of known classes. This method is an unsupervised learning method, which has been applied to image feature extraction [2]. Visual $\mathrm{C}++$ is one of the most popular software development tools today. The graphic design of AI combines the technical essence of Microsoft. In particular, its advantages in compiler optimization technology make it more suitable for the development of image processing software [3]. However, these algorithms are based on the RGB color space of pictures. For pictures with color illusion, the extracted color value is significantly different from the color observed by human eyes, which is not in line with people's intuitive feeling.

In real life, the color in color images is mainly described by semantics. In digital devices such as computers, the representation of image color is based on data domain. Color image provides people with rich and colorful information, and the regions of interest in the image can be extracted through the regional characteristics of gray, texture and color. Therefore, this paper skillfully uses the transformation of image color space to propose an automatic color extraction algorithm of graphic design image based on artificial intelligence.

\section{AUtOMATIC COLOR EXTRACTION ALGORITHM FOR \\ SURFACE DESIGN IMAGE}

The conversion between color space and various color spaces is a very important problem before color feature extraction of graphic design images. In the color feature representation of color image, there are many color spaces to choose, and the choice of color space has an impact on the final result of image retrieval. In this paper, HSI space is selected for feature extraction, and on this basis, the color feature set of graphic design image is constructed. Finally, the automatic image color extraction is realized by artificial intelligence algorithm.

\section{A. Color space characteristics of graphic design} image

In order to make all kinds of colors can be arranged in a certain order and contained in a space, the three-dimensional coordinate axis is corresponding to the three independent parameters of color, so that each color has a corresponding 
space position. Conversely, any point in the space represents a specific color, and this space is called color space. The quantitative representation of image color information is completed in color space (color coordinate system). Color space is three-dimensional [4]. As the three-dimensional coordinates of color space, the three independent parameters can be the three psychological attributes of color: hue, saturation and intensity. They can also be the other three parameters, such as RGB, lab or CMY. As long as the three parameters describing color are independent of each other, they can be used as the three-dimensional coordinates of color space. CMYK color space, YCC color space, CIE color space, RGB color space and HSI color space are commonly used [5]. The RGB color coordinate method takes red $\mathrm{R}$, green $\mathrm{G}$ and blue $\mathrm{B}$ as the primary colors, and the other colors are weighted and mixed by these three primary colors. RGB color space is a common color space, because most digital images are expressed in this color space.
However, the main disadvantage of RGB space is that it is not intuitive. It is difficult to know the perceptual attribute of the color described by the RGB value. Secondly, RGB color space is one of the most uneven color spaces, and the difference between two colors cannot be expressed as the distance between two points in the corresponding color space. If the RGB value is converted into hue, color saturation and brightness, the problem is solved [6]. Therefore, some other color spaces which are more in line with visual perception are proposed. HSI is more commonly used, and its three components represent hue, saturation and brightness respectively. In HSI model, $\mathrm{H}$ component is the most similar to human vision in describing color. In many applications, when the color image is transformed from RGB space to HSI space for retrieval, only $\mathrm{H}$ component can be considered to simplify the operation and improve the processing speed. As is shown in Figure 1.
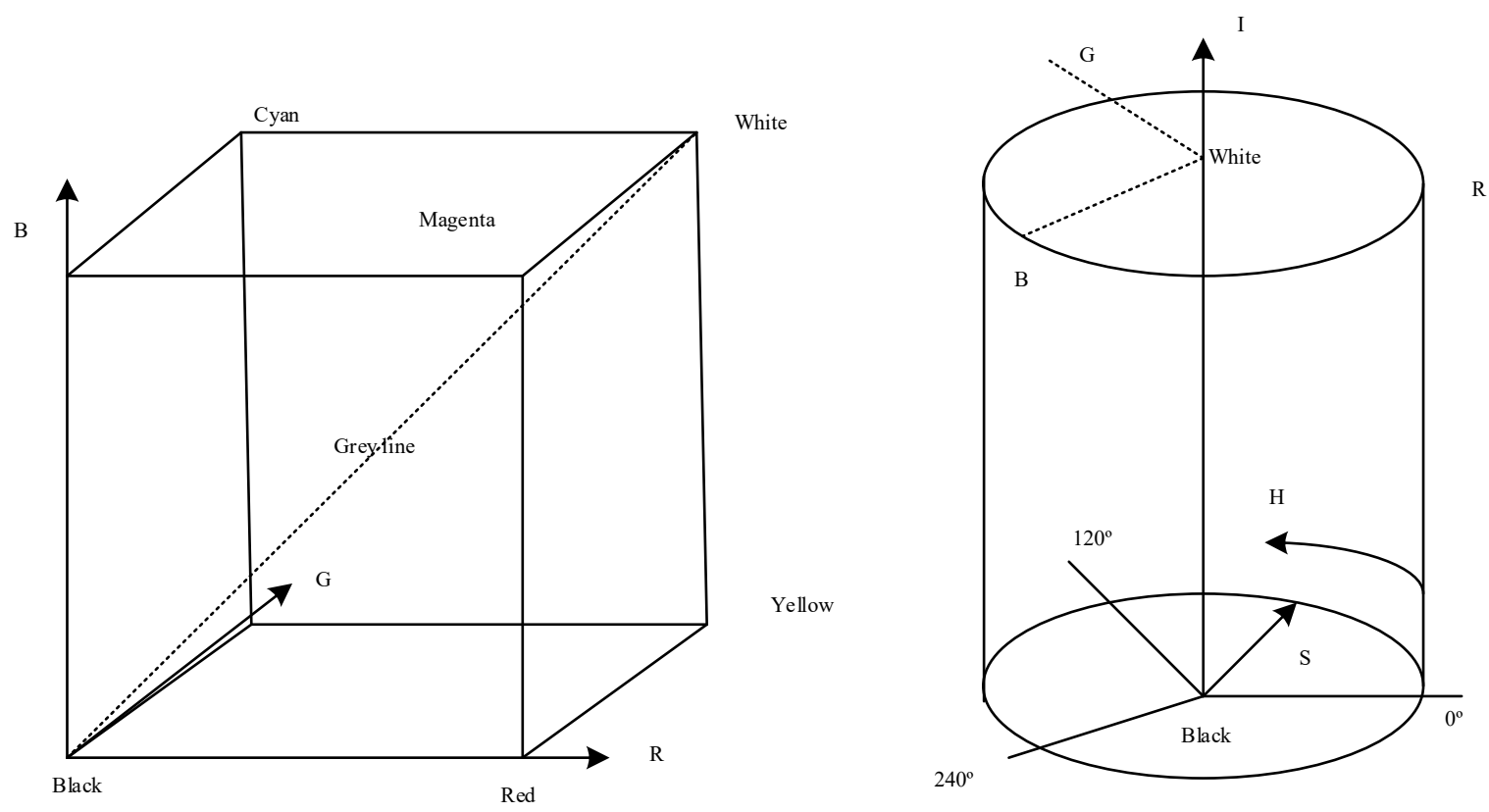

Figure 1. Two color space coordinate systems

RGB color space is the most common color space. RGB color space is based on the color space of color adding model of color science [7]. The so-called color adding model is relative to the color reduction model. In addition to the original color, the color in the color adding model can be mixed by proportion of the original color, while the subtraction model considers that any color is obtained by subtracting a certain proportion of the original color components from the pure white [8]. There is no contradiction between color adding and color reducing models, which reflects the understanding of color composition. The most common color reduction space is CMYK space. For RGB space, the primary colors are red (wavelength $700 \mathrm{~nm}$ ), green (wavelength $546.1 \mathrm{~nm}$ ) and blue (wavelength $435.8 \mathrm{~nm}$ ). The color space is constructed by using three-dimensional coordinate system [9]. The three axes of coordinate system are R, G, B components, each component has the strength of 0 to 255 , and the zero intensity of all primary colors is black, and the strongest brightness of all primary colors becomes white. A set of ( $R$, $\mathrm{G}, \mathrm{B})$ coordinates can be used to define any color in the space [10]. RGB color space is the most common color format to store images in digital imaging. The data pictures processed are RGB pictures. Conversion formula from RGB format to HSI format:

$$
\begin{aligned}
& H=\operatorname{ang} \frac{1}{\sqrt{6}}(2 R-G-B) \frac{1}{\sqrt{2}}(G-B) \\
& S=\sqrt{\left(4 R^{2}+4 G^{2}+4 B^{2}-2 R G-2 R B-2 G B\right) / 6} \\
& I=\frac{1}{\sqrt{3}}(R+G+B)
\end{aligned}
$$

The model calculation based on image structure similarity is successful, considering the large number of distortion types that can damage the image structure information, and the human visual approach is highly sensitive to the structure [11]. The SSIM extracts the brightness, contrast, and structural features, calculates the SSIM index based on 
the reference and distortion images, and then summarizes the fusion features to obtain the mass score. SSIM expression:

$$
\operatorname{SSIM}(x, y)=\frac{\left(2 \mu_{x} \mu_{y}+c_{1}\right)\left(2 \sigma_{x y}+c_{2}\right)}{\left(\mu_{x}^{2}+\mu_{y}^{2}+c_{1}\right)\left(\sigma_{x}^{2}+\sigma_{y}^{2}+c_{2}\right)}
$$

Where $\mu_{y}$ is contrast and structural similarity, $\mu_{x}$ and $y$ are two pictures, $\sigma$ is a constant. Gradient feature is also an important feature for image structure and sensitive to human eyes. Based on this, FSIM model first extracts the phase consistency and gradient amplitude of the image, and then combines them into the following formula to define a structural similarity parameter:

$$
s(x, y)=\frac{2 f_{x} f_{y}+\mathrm{a}}{\operatorname{SSIM}(x, y)\left[f_{x}^{2}+f_{y}^{2}\right]+\mathrm{a}}
$$

Where a represents the phase consistency or gradient amplitude characteristics, and a is a stability factor [12]. The image acquisition module is mainly composed of FPGA controller, image sensor, memory SDRAM and Serial UART. FPGA controller is an important part of image acquisition module. The overall structure of the module is shown in the Figure 2.

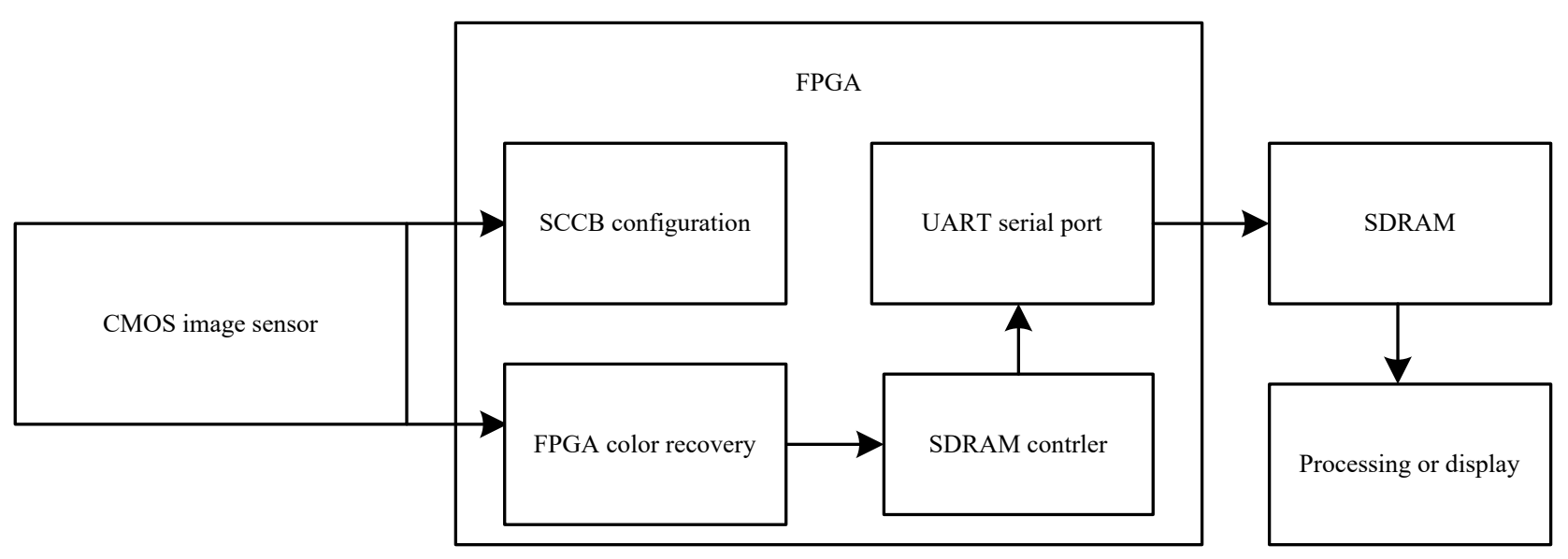

Figure 2. Overall structure of image acquisition module

In addition to the study of the structure and signal characteristics of the image, the image aesthetic quality evaluation needs to study the image composition, color, exposure, content and other high-level information [13]. Considering the factors of color, texture, shape, size, depth of field, three-way rule and regional contrast, a picture quality classifier is trained. The features of picture simplicity, clarity, color, contrast and average brightness (exposure) are extracted to classify professional and amateur photos [14]. The color harmony model is introduced into the study. The deep neural network is used to solve this problem. The quality classification of images is done by hand extracting features and a machine learning algorithm. Because of the many aspects of image aesthetics and complex problems, the current algorithm effect is far from meeting the application requirements [15]. The image information is extracted by the image acquisition module and transmitted to the image color enhancement module. The image color enhancement module uses the color image enhancement algorithm based on visual characteristics to adjust the overall brightness of the image and enhance the local contrast, and completes the color enhancement output of the plane vision image to the artificial intelligence interface to the user.

\section{B. Color feature collection of graphic design image}

After the image information is collected by the image acquisition module, the information is transmitted to the image color enhancement module. When the collected image is full of a row of effective pixels in the SDRAM memory, the image data of this row is migrated to the image acquisition storage area of the external memory DDR [16]. When the image data in this storage area is larger than 1 frame, the image color enhancement module uses the color image enhancement algorithm based on visual characteristics to adjust the overall brightness of the image and enhance the local contrast. The processed image data is copied to the DDR image revisit storage area, and then the image data in this area is transferred to the FIFO of image output [17]. The standard image is displayed by DE90CF383 coding, and the plane vision image color enhancement processing of AI interface is completed. The image color enhancement module is shown in the Figure 3. 


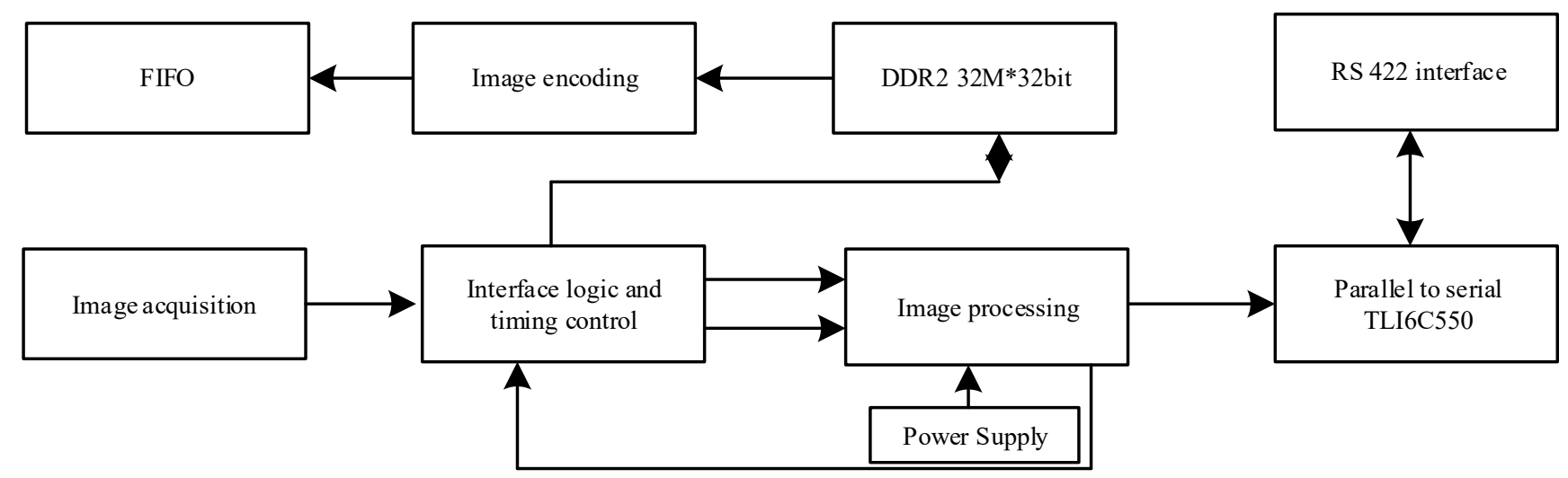

Figure 3. Structure of image color enhancement module

The traditional image color enhancement module uses the color image enhancement algorithm based on visual characteristics to adjust the overall brightness of the image. At the same time, the overall brightness adjustment of the plane visual image to enhance the local contrast is mainly based on the nonlinear adaptive pull-up of the histogram to realize the enhancement of the dark area in the interface [18]. CMYK color model is a color model composed of complementary colors of red, green and blue, cyan, magenta and yellow. Different from the display, it is a combination of subtractive colors by printing the reflected light of color ink and color paint. The color model composed of cyan, red and yellow is equivalent to subtracting a certain color from white light, so it is also called the subtraction method. In Cartesian coordinate system, the appearance of CMY color model is similar to that of RGB color model, but the origin and vertex are just opposite [19]. The origin of CMY model is white, and the corresponding vertex is black. The simple conversion from CMY to RGB is as follows:

$$
\left\{\begin{array}{l}
R=1-C \\
G=1-M \\
B=1-Y
\end{array}\right.
$$

HIS color space and RGB color space are different representations of the same physical quantity. For any three RGB values normalized to $[0,1]$, they can be converted to HSI model. The H, S, I components of this model can be calculated by the following formula:

$$
\left\{\begin{array}{l}
\mathrm{H}=\arctan (\sqrt{3}(\mathrm{G}-\mathrm{B}) /(\mathrm{R}-\mathrm{G})+(\mathrm{R}-\mathrm{B}) \\
\mathrm{I}=(\mathrm{R}+\mathrm{G}+\mathrm{B}) / 3 \\
\mathrm{~S}=1-\min (\mathrm{R}, \mathrm{G}, \mathrm{B}) / \mathrm{I}
\end{array}\right.
$$

In HSI space, the distance measure between two colors $C_{i}$ and $\mathrm{C}_{\mathrm{j}}$ are defined as:

$$
\mathrm{D}\left(\mathrm{C}_{\mathrm{i}}\right)=\left[\left(\mathrm{I}_{\mathrm{i}}+\mathrm{I}_{\mathrm{j}}\right)^{2}+\left(\begin{array}{l}
\mathrm{S}_{\mathrm{i}}\left(\cos \mathrm{H}_{\mathrm{i}}-\mathrm{S}_{9}^{\mathrm{co}} \mathrm{H}_{\mathrm{j}}\right)^{2}+ \\
\left(\mathrm{S}_{1} \operatorname{sirH}_{\mathrm{i}}-\mathrm{S}_{j} \mathrm{snH}_{\mathrm{j}}\right)^{2}
\end{array}\right]^{1 / 2}\right.
$$

Where:

$$
\cos H=\frac{2 \mathrm{D}\left(\mathrm{C}_{\mathrm{i}}\right)}{2 \sqrt{(\mathrm{R}-\mathrm{G})^{2}+(\mathrm{R}-\mathrm{B})(\mathrm{G}-\mathrm{B})}}
$$

The most basic representative models based on space and feature are root mean square error (MSE) and peak signal-to-noise ratio (PSNR). Without considering the characteristics of human visual methods, it is simple to measure the difference between two images from the angle of signal, but it still has a lot of value [20]. HSI model has its unique advantages in many processing. In his model, the luminance component is separated from the chroma component, and the component has nothing to do with the color information of the image. Secondly, in his model, the concepts of hue $\mathrm{H}$ and saturation $\mathrm{S}$ are independent of each other and closely related to human perception. These characteristics make his model very suitable for color perception based on human vision. Set the luminance component $\operatorname{MaxRGB}(i, j)$ of the plane vision image as the maximum value of the three primary colors $R, G, B$, as shown below:

$$
\begin{aligned}
& \operatorname{MaxRGB}(i, j)=\cos \mathrm{H}- \\
& \max (\text { Ori } R(i, j), \text { Ori } G(i, j), \text { Ori } B(i, j))
\end{aligned}
$$

In the formula, the R, G and B components of the pixel ( $\mathrm{i}$, $j)$ of the original image in RGB space are represented by Ori $R(i, j)$, Ori $G(i, j)$ and Ori $B(i, j)$ in turn. The number of pixels in $\operatorname{MaxRGB}(i, j)$ exceeds the threshold $\omega$. The number is described by $\mathrm{m}$, and the threshold is $0 \omega$ by:

$$
\omega=\operatorname{uint} 8\left(\operatorname{Long} \times \mathrm{W} \times \frac{100}{256}\right) \operatorname{MaxRGB}(i, j)
$$

The length and width of the image are set as $\omega$, width, 256 as the number of gray values, and 100 as the amount of threshold. The gray values of pixels not larger than the threshold need not be sorted out to reduce the interference of a small number of gray values on the mapping:

$$
\operatorname{TraRGB}(i, j)=255((n-1) /(m-2)) g \omega
$$

Where: $\mathrm{n}$ and $\mathrm{m}$ are constants, and the value range is $[0$, $\left.\mathrm{m}^{-1}\right]$; $\operatorname{TraRGB}(i, j)$ is the gray value after mapping, and the best gray value $g_{1}$ of image pixel is:

$$
\begin{aligned}
& g_{1}=0.0439 \cdot(\operatorname{In}(\text { ValueMean }))^{2}- \\
& 0.1163 \operatorname{In}(\text { ValueMean })+0.5733 \operatorname{TraRGB}(i, j)
\end{aligned}
$$

After the overall brightness adjustment, the details of the 
overall low illumination area of the image can be enhanced. Next, we need to enhance the local contrast of the image brightness through the correlation between the gray values of the local area pixels to make the image details more significant [21]. The calculation window size is $9 \times$ the median filter can store the edge details of the image completely. So median filter is used to calculate the mean value of brightness in this range:

$$
\operatorname{MedRGB}(i, j)=g_{1} \operatorname{median}_{t}(\operatorname{TraRGB}(i, j))
$$

After obtaining the average brightness by formula, the local contrast of image color is enhanced by formula:

$$
\begin{aligned}
& \operatorname{ResRGB}(i, j)=g_{2}(\operatorname{TraRgB}(i, j) \\
& -\operatorname{MedRGB}(i, j))+\operatorname{TraRgB}(x, y)
\end{aligned}
$$

Where: $g_{2}=2 ; \operatorname{TraRGB}(i, j)$ is the gray value of the pixel after the overall brightness processing; $\operatorname{TraRGB}(i, j)$ is the average value of regional brightness; $\operatorname{ResRGB}(i, j)$ is the gray value of local contrast enhancement. After repeated experiments, the following parameters were found to quantify the difference, including correlation coefficient:

$$
\begin{aligned}
& \frac{d\left(H_{R}, H_{D}\right)=}{\sum_{I}\left(H_{R}(I)-\bar{H}_{R}\right)\left(H_{D}(I)-\bar{H}_{D}\right)} \\
& \operatorname{ResRGB}(i, j) \sqrt{\sum_{I}\left(H_{R}(I)-\bar{H}_{R}\right)^{2} \sum_{I}\left(H_{D}(I)-\bar{H}_{D}\right)^{2}}
\end{aligned}
$$

Contrast plays an important role in image perception. Based on the recently discovered free energy principle, the free energy theory is effective in quality evaluation, especially in the image analysis of contrast change. Therefore, the information $d i$ theory is introduced as the consideration of image contrast:

$$
E(x)=-\sum_{i=0}^{255} P_{i}(x) \log P_{i}(x)
$$

Where $P_{i}$ the probability and the base of $\log$ is 2 . The difference between the two graphs is the characteristic:

$$
d_{E}=d\left(H_{R}, H_{D}\right) / E_{R}-E_{D} E(x)
$$

In addition to the above features, hue number has been

\begin{tabular}{|c|c|c|}
\hline Category & Feature name & Characteristic meaning \\
\hline \multirow{6}{*}{ Basic color features } & $\mathrm{V}_{\mathrm{V}}$ & Luminance variance \\
\hline & $\mathrm{V}_{\mathrm{S}}$ & Saturation variance \\
\hline & $\mathrm{V}$ & Average brightness \\
\hline & $\mathrm{V}_{\mathrm{H}}$ & Hue variance \\
\hline & $\mathrm{H}$ & Average hue \\
\hline & $\mathrm{S}$ & Average saturation \\
\hline \multirow{2}{*}{ Color edge features } & $\mathrm{D}^{\mathrm{L}}(\mathrm{R}, \mathrm{D})$ & Edge difference of channel L \\
\hline & $\mathrm{D}^{\mathrm{b}}(\mathrm{R}, \mathrm{D})$ & Edge difference of channel B \\
\hline $\begin{array}{l}\text { HSVSpatial distribution } \\
\text { Characteristics }\end{array}$ & $\mathrm{C}$ & Correlation coefficient of HSV histogram \\
\hline Other features & $\mathrm{G}$ & Image softness is the average gradient of the image \\
\hline
\end{tabular}
proved to be a useful feature in the study of image authenticity prediction [22]. Image softness, that is, the average gradient of image, is also often mentioned in photo professional prediction. In addition, as a classic and efficient quality evaluation index, PSNR is also used in this paper. As is shown in Table 1.

Table 1. Summary of extracted features

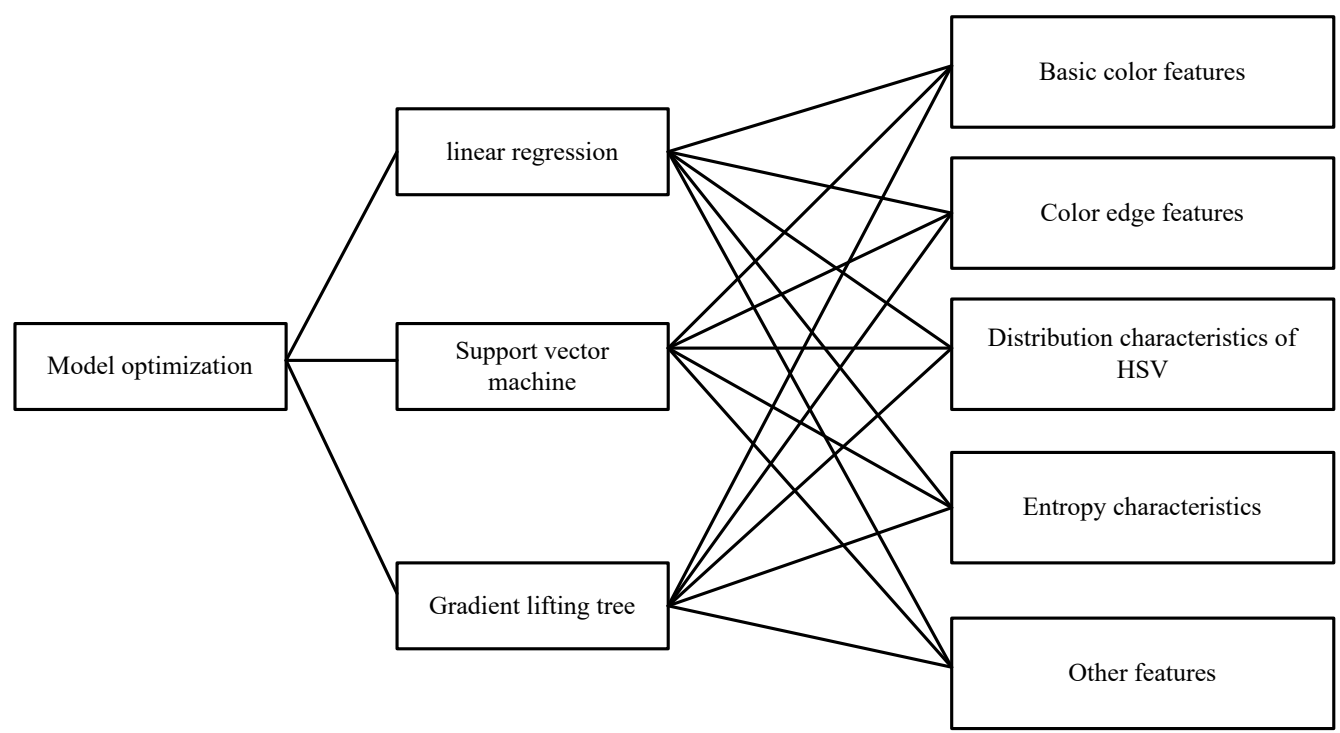


Figure 4. Algorithm path diagram of quality evaluation based on image color difference features

According to the extracted features shown in Table 1, the quality of image color change is evaluated by using characteristic elements such as brightness, saturation, hue, channel edge difference and image softness. Through the above analysis, it can be seen that in order to better extract the color of graphic design image, more image color feature information is required. Therefore, this paper attempts to extract a large number of features related to color change to form a feature library, and then use various machine learning regression algorithms to train the model and establish a subjective database data suitable for image color change. In order to get the best model algorithm, the training adopts the methods of feature normalization, cross validation and grid search. Then the correlation analysis of each feature is carried out, and the meaningful features are selected to prove the significance of color correlation features in color change image quality evaluation. The research path is shown in the Figure 4.

The creation of image database has clear relevant standards and industry practices that can be followed, which is generally divided into four steps: image collection, image processing, subjective evaluation and analysis, as shown in the Figure 5.

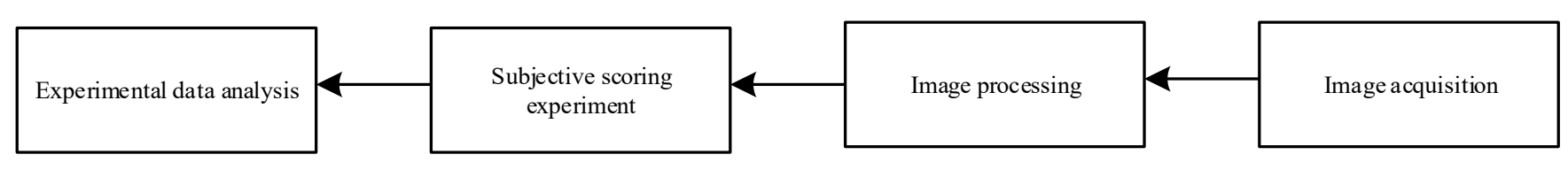

Figure 5. Flow chart of database creation

Image collection uses computer simulation method to generate distorted images, only reference images need to be collected. If you plan to use real distorted images, in addition to the reference images, the distorted images should also be collected. The collected images need to meet the needs of research purposes. Firstly, they must be of high quality. Secondly, they must be selected for image content. The images are rich in color and clear in theme. If it is a general database, it is necessary to make the reference image content as wide as possible, and the number of each content category is relatively average. Image processing includes image standardization and distortion generation. Standardization processing refers to the operation of image cutting and naming, while distortion image generation uses algorithm simulation method to generate multiple versions of images that need distortion types, and there should be certain distortion scale differences between multiple versions.

\section{Realization of automatic extraction of image color}

Image segmentation is an important problem in image processing and computer vision. Its purpose is to divide an image into different regions with different characteristics, and provide necessary information for further analysis, recognition, recognition and compression of images. For color image segmentation, two problems are mainly solved, one is the appropriate color space selection, and the other is the segmentation method. Color space has been through a long time since it was proposed. Most of them are made locally or specially used in a certain field. RGB, HSV, his, CMYK and so on are commonly used. The appropriate color space can be selected according to different requirements. For segmentation methods, so far, a lot of color image segmentation algorithms have been proposed. Most of the algorithms are gray image segmentation methods directly applied to color space. These image segmentation methods are mainly based on feature information and spatial information. In feature-based methods, image segmentation is considered as an application of pattern recognition, which can be further divided into supervised classification and unsupervised classification. Because of the fuzziness and inhomogeneity of the image itself, the fuzzy theory has the ability to describe the fuzzy problem of image, and the segmentation result should conform to the human sensory and visual characteristics. So it is necessary and reasonable to use fuzzy theory to process information technology and apply it to image processing. Image semantics is an abstract expression of human understanding of image content, and is essentially a representation of knowledge. As mentioned above, the content of the images is very rich. Because of the existence of individual differences, people must have subjectivity in understanding images. Therefore, the understanding of an image is different from different cognitive subjects and different perspectives. How to effectively express the semantic information of an image is an important aspect of image semantic research. The content or information contained in the image is very rich. The semantic description of the image is analyzed based on different semantic levels. The scope or granularity of the semantic description at different levels is different. The classification of semantic level of image is closely related to the classification of image feature structure. In the early stage of image research, according to the physical characteristics of images, images are only regarded as the collection of underlying visual features. And the classification of semantic level is also developing with the deepening of image understanding. As is shown in Figure 6.

Color feature extraction based on semantics is a method of color feature extraction using fuzzy clustering algorithm. The main process of extraction is as follows: firstly, the color image is read in, which is automatically converted into 8-bit RGB format according to different image formats, and the image is scaled to the appropriate size at the same time; Then, the color features of the image to be segmented are identified, and the color features to be segmented are extracted, and compared with the semantic color samples in the database, and the corresponding semantic color is found; Then, according to the selected target color as the clustering center of image segmentation, fuzzy clustering algorithm is used for clustering analysis to intercept the appropriate color partition; Finally, the results of extraction and segmentation 
are analyzed. The process of color feature extraction can be simply illustrated by the following block diagram. The extraction process of semantic color features of an image is mainly composed of several modules, such as image data reading, pixel matrix establishment, data processing, clustering, feature extraction and selection, as shown in the Figure 7.

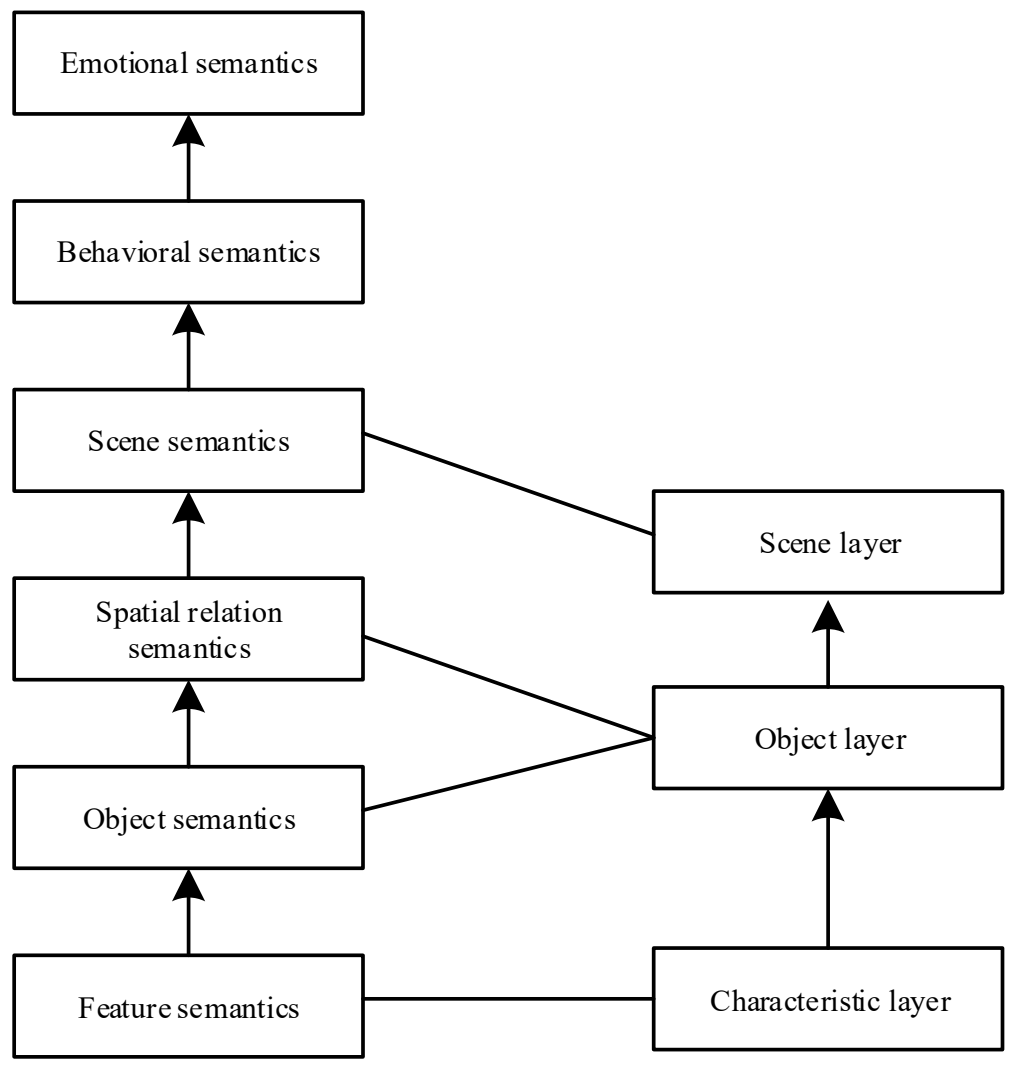

Figure 6. Semantic hierarchy model of image

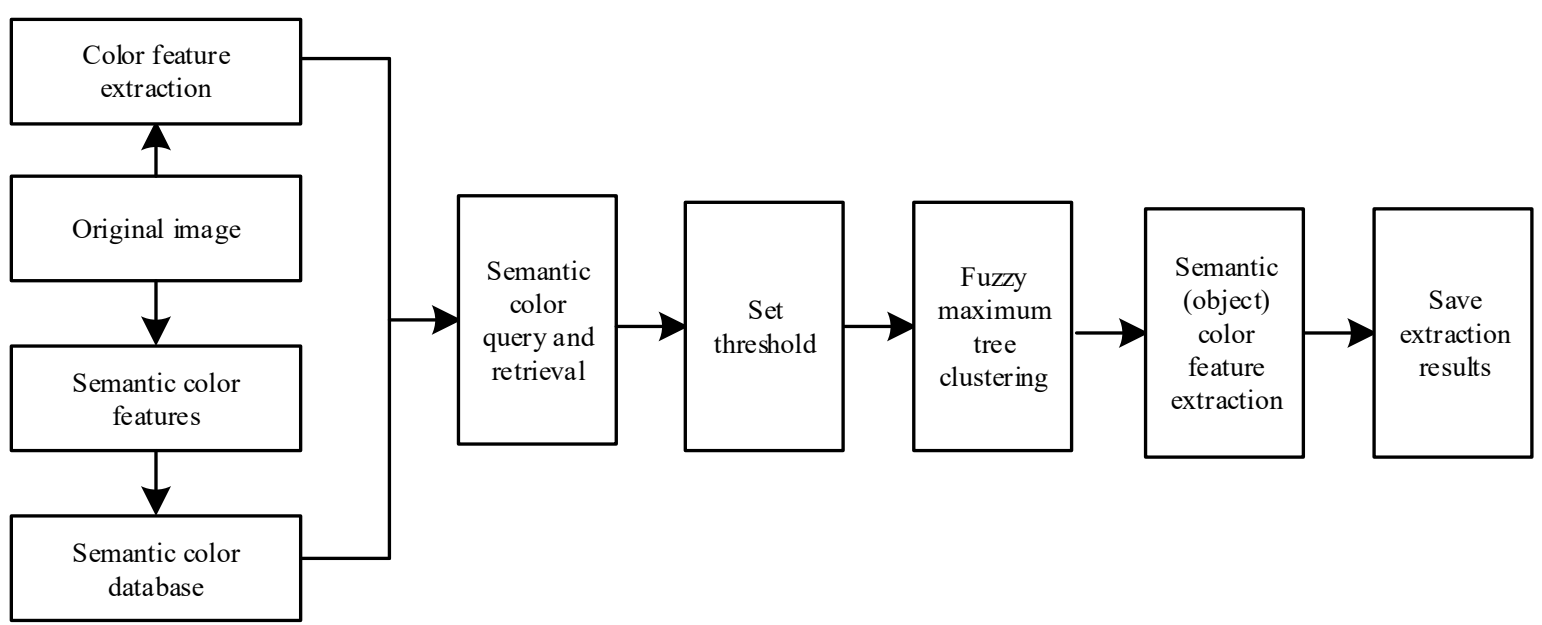

Figure 7. Image data feature extraction and selection module

Based on the three-layer structure of image, the simplest level division is to divide the image semantics into three structural levels. The first layer is the feature semantic layer, which describes the underlying physical features of the image, such as color, texture, shape, edge and so on, which is the basis of content-based image analysis. The second layer involves the high-level features and their semantic description derived from the reasoning and analysis of low-level visual features; the third layer is the higher-level semantics, including scene semantics, behavior semantics and emotion semantics, obtained by further reasoning of objects and spatial relations. In essence, the feature layer does not correspond to the real image semantics, because it is based on the physical characteristics of the image, and does not add human understanding and abstraction. In the past, the analysis and processing of images mostly focused on this level. Using text information to describe image or image region is a popular way. Although text-based semantic description is intuitive and easy to deal with abstract objects, it needs a lot of manual annotation, cannot 
automatically obtain the text description of image content, and has limited ability to express the complex relationship between concepts. In recent years, through the introduction of computer linguistics and other information processing methods, the semantic representation method based on text expression has better fuzzy matching ability, and the expression ability has been enhanced. The reason why color harmony models are contradictory and can not be integrated is that the existing color harmony models are designed based on rules. The essence of the color harmony model is to study the relationship between color and color. Based on this idea, it can be assumed that between any two colors in nature there is a value representing their subjective feelings, which becomes a harmonious relationship. The color harmony model is to fit the whole harmonious relationship. Due to the complexity of human visual methods and the subjectivity of personal views, the rule design is naturally immature, so the effect of traditional artificial design model is poor. For this problem, we adopt the modern concept of data learning to solve. Although there are differences in each person's views, a large number of people can get opinions that can represent the mainstream views. The color harmony relationship is the same. The color harmony model can be obtained by learning and training a large number of data. When the amount of data is large enough and the method is reasonable, the universal color harmony relationship can be obtained. The knowledge based on data is more convincing than the traditional model based on rules. The specific method is to assume that the color quality score of an image is determined by the relationship between its main colors. Then, to extract the main color of a picture and establish a numerical relationship between the image quality score and the harmonious relationship between the main color pairs, the color harmonious relationship can be acquired according to the image subjective quality score, and the image quality score can also be predicted according to the existing color harmonious relationship. The relationship between the image quality score and color harmony is that the spatial characteristics of the image must be considered, that is, the position and importance of color in the image. Therefore, this paper introduces the image saliency calculation technology. Learning the knowledge of color harmony from a large amount of image data can solve the problem that the traditional color harmony model is contradictory and cannot be combined. Moreover, the traditional color harmony model is not suitable for complex colors and cannot perform regression calculation. For example, in order to fully reproduce on various display devices, such as monitors, printers and handheld devices, Color images should be processed according to the color gamut of the target device. This process follows the gamut mapping mechanism. When an image is transmitted from one device to another, it will convert each color outside the gamut into the closest reproduction color in the image data. However, in this process, the color outside the color gamut is usually mapped to the boundary point of the target color gamut. Often, different color points are mapped to the same boundary point of the target color gamut, resulting in the loss of a large amount of color information in the original image, resulting in blur and halo noise in the reproduced color image. The above phenomena will lead to large deviation of the regression model. Therefore, the knowledge of image saliency is introduced to consider the spatial distribution of color. The technical path is shown in the Figure 8.

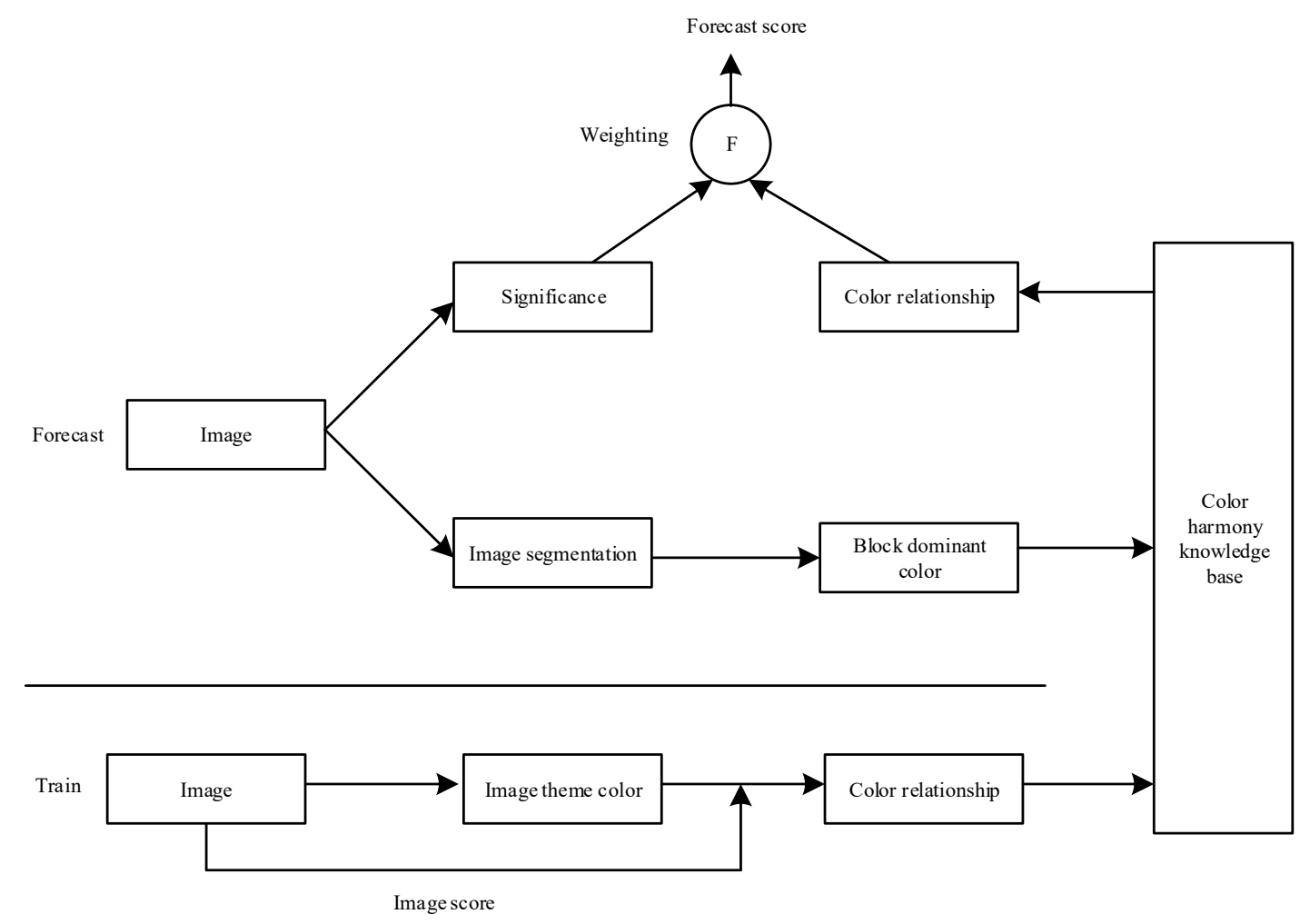

Figure 8. Flow chart of image quality evaluation method based on color harmony relationship learning

The framework is divided into two parts: training and prediction. In the training part, the information of color 
harmony is extracted through image processing, that is, the numerical relationship between the theme colors of each image, which is persistent to the database. As the knowledge of color harmony, in the process of a large amount of data training, the database will be constantly updated and improved. In the prediction phase, for an input image, color block segmentation and saliency calculation are performed respectively. As one of the important characteristics that determine the quality of color image, color harmony has attracted the attention of a large number of researchers. For a simple color distribution, its harmony can be judged by calculating the relative differences of hue, saturation and brightness between the two colors. In this paper, the main color of the local area of the image block is used to represent the color harmony of the image by calculating the relative differences between the hue, saturation and brightness of the local image block and the main color. Firstly, the local image block is converted from RGB color space to Munsell color space, the main color of the image block is calculated according to the hue value, then the hue value of each pixel in the image block is subtracted from the main color to obtain the absolute value, and then their frequency distribution histogram is calculated. Finally, the color harmonic library is queried according to the color block segmentation results, The harmony score between color blocks is obtained, so as to obtain higher quality graphic design image color extraction results.

\section{ANALYSIS OF EXPERIMENTAL RESULTS}

In order to verify the effectiveness of this method, this paper compares the color enhancement effect of plane vision image based on design artificial intelligence interface with the original image, and implements it with Visual $\mathrm{C}++6.0$ programming language. In order to test the efficiency of the algorithm for content-based image retrieval database, 500 color images are selected and color features are extracted from the images, under the same test conditions (i.e. select the same case image and the same similarity), check the time of the returned result and the accuracy of the returned image. The results compared with the sequential retrieval algorithm are shown in the Table 2.

Table 2. Image retrieval test results

\begin{tabular}{ccccc}
\hline & $\begin{array}{c}\text { Retrieved } \\
\text { image }\end{array}$ & $\begin{array}{c}\text { Related } \\
\text { images }\end{array}$ & $\begin{array}{c}\text { Retrieval } \\
\text { time }\end{array}$ & $\begin{array}{c}\text { Precision } \\
\text { ratio }\end{array}$ \\
\hline $\begin{array}{c}\text { Traditional } \\
\text { methods }\end{array}$ & 13 & 3 & 102 & $96 \%$ \\
$\begin{array}{c}\text { Method of } \\
\text { this paper }\end{array}$ & 29 & 4 & 305 & $95 \%$ \\
\hline
\end{tabular}

In order to fully prove the effectiveness of this method, the histogram of the plane vision image using this method is compared, and the comparison results are shown in Figures 9-10.

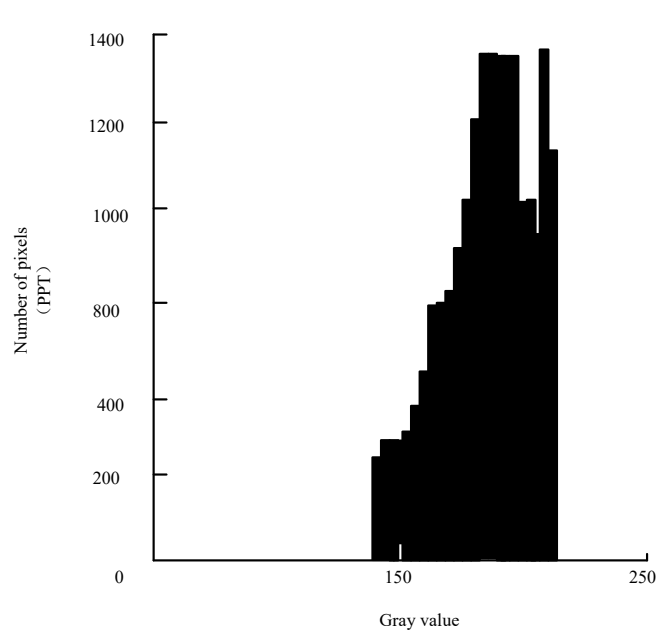

Figure 9. Original RGB histogram

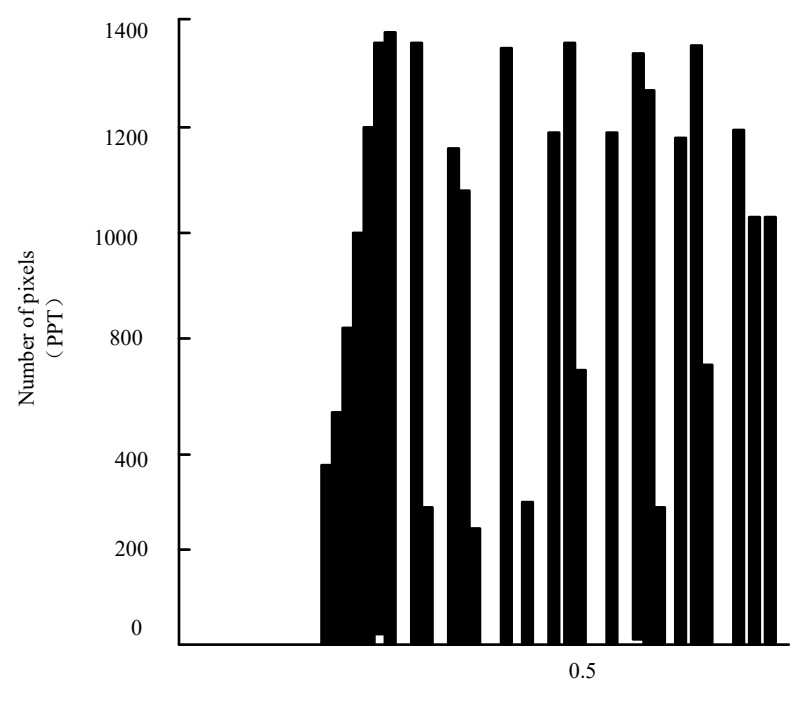

Gray value

Figure 10. Histogram of enhanced RGB image

Table 3. Summary of existing subjective quality evaluation

\begin{tabular}{|c|c|c|c|c|}
\hline Name & $\begin{array}{l}\text { Number } \\
\text { of } \\
\text { reference } \\
\text { pictures }\end{array}$ & $\begin{array}{c}\text { Total } \\
\text { number } \\
\text { of } \\
\text { pictures }\end{array}$ & $\begin{array}{l}\text { Number } \\
\text { of } \\
\text { distortion } \\
\text { types }\end{array}$ & $\begin{array}{l}\text { Types of } \\
\text { color } \\
\text { dependent } \\
\text { distortion }\end{array}$ \\
\hline TID2019 & 25 & 1700 & 17 & $\begin{array}{c}\text { Color } \\
\text { additive } \\
\text { noise }\end{array}$ \\
\hline LIVE & 29 & 779 & 5 & Nothing \\
\hline CSIQ & 30 & 866 & 6 & Nothing \\
\hline TID2020 & 25 & 3000 & 24 & $\begin{array}{c}\text { Saturation } \\
\text { change }\end{array}$ \\
\hline MICT & 14 & 168 & 2 & Nothing \\
\hline A57 & 3 & 54 & 6 & Nothing \\
\hline
\end{tabular}

According to the analysis of the image, the original RGB gray value is relatively concentrated, and the details in the side reflection of the original image are fuzzy, the contrast is not high, and the contour significance is low. In addition to the above image database for general quality evaluation, there are also other databases related to color changes. 
However, due to different research focuses, none of them can meet the needs of the market. A database containing 5000 original images and 5 different color versions of each image has been released. As in shown in Table 3.

After using this method, the gray value distribution is more uniform, which shows that the enhanced image has significant details, clear edges and better plane vision than the original image. This method can improve the clarity of image color. In the experiment, the average gray value and contrast enhancement index (R) of octree algorithm, clustering algorithm and this algorithm mentioned in the introduction are calculated, and compared with the two evaluation indexes of overall brightness and local contrast. The performance results of these three methods are shown in Table 4.

Table 4 Enhancement effect of three algorithms

\begin{tabular}{ccccccc}
\hline & \multicolumn{3}{c}{ Octree } & \multicolumn{2}{c}{$\begin{array}{c}\text { Clustering } \\
\text { algorithm }\end{array}$} & \multicolumn{2}{c}{$\begin{array}{c}\text { The paper } \\
\text { method } \\
\text { Inde } \\
\mathrm{x}\end{array}$} & $\begin{array}{c}\text { Over } \\
\text { all } \\
\text { brigh } \\
\text { tness }\end{array}$ & $\begin{array}{c}\text { Local } \\
\text { contras } \\
\mathrm{t}\end{array}$ & $\begin{array}{c}\text { Overa } \\
\text { ll } \\
\text { bright } \\
\text { ness }\end{array}$ & $\begin{array}{c}\text { Local } \\
\text { contra } \\
\text { st }\end{array}$ & $\begin{array}{c}\text { Overa } \\
\text { bright } \\
\text { ness }\end{array}$ & $\begin{array}{c}\text { Local } \\
\text { contra } \\
\text { st }\end{array}$ \\
\hline $\mathrm{R}$ & 3.23 & 100.32 & 1.12 & 95.23 & $\begin{array}{c}174.2 \\
\text { Mea } \\
\mathrm{n}\end{array}$ \\
$\begin{array}{c}\text { valu } \\
\text { e of } \\
\text { gray } \\
\text { valu } \\
\text { e }\end{array}$ & 139.4 & 3.21 & 120.4 & 10.11 & 3 \\
\hline
\end{tabular}

By analyzing the data in Table 4 and analyzing the gray value mean data from the two aspects of overall brightness and local contrast, it can be seen that the gray value mean of this method is 174.23110 .23 , which is greater than that of the other two methods. The $\mathrm{R}$ values of this method are 4.32 and 4.65, which are also larger than the other two methods. Experimental results show that this method can effectively improve the overall brightness and contrast of the image, and the enhancement effect is better than octree algorithm and clustering algorithm.

\section{DISCUSSION}

Color images affect human life style in many ways. For example, people share color pictures with family and friends through various mobile phone clients, which makes the original simple text or gray-scale picture communication no longer boring. However, when a color image is transmitted to the human eye, it has gone through many intermediate stages, including image acquisition, processing, compression, storage, transmission and reproduction. Because color images are stored digitally, the change of any one or a group of pixels will greatly change the visual characteristics and quality perception of the image. At the same time, the probability of color image distortion is very high, so it is very important to design quality evaluation algorithm for color image. The quality evaluation of color image can not only detect the integrity of the image, but also optimize the image parameters to produce the best visual feeling. Therefore, this paper is committed to designing a color image quality evaluation algorithm with high consistency with human perception by extracting color features that can effectively represent color distortion from the perspective of color frequency domain features, redefinition of color space and color visual characteristics.

\section{CONCLUSIONS}

Color is one of the most direct and important features of human recognition of objects. Human beings can naturally recognize colors in different situations, but computer extracted colors lack the recognition of situations. They are easily affected by physical surface texture, illumination and shadow. They can not accurately recognize the same color in different environments, so they can only simply extract its RGB spatial value, However, RBG values cannot be mapped to the corresponding color category, and it is not understood that two different RGB values belong to the same color category.

The color feature extraction algorithm skillfully uses the transformation of color space to realize the basic operation of image preprocessing, and realizes the automatic extraction of color features through appropriate data clustering algorithm. Finally, an example is given to verify the effectiveness of the method and the practicability of the software. Compared with the color extraction method based on RGB color space, this method has the following advantages: (1) It is closer to people's intuitive visual experience; (2) The complex RGB color values are classified according to the Chinese color naming rules; (3) It solves the problem that a small number of color illusion pictures cannot extract color by traditional methods. Thus, it lays a foundation for the automation of the whole method and the next research.

\section{REFERENCES}

[1] R. Dev and N. K. Verma, "Robust noisiness measure based improved generalized fuzzy peer group for removal of mixed noise from color image", IEEE Signal Processing Letters, vol. 26, no. 2, pp. 267-271, 2019.

[2] A. Othman, T. Wook and F. Qamar, "Categorizing color appearances of image scenes based on human color perception for image retrieval", IEEE Access, vol. 8, pp. 161692-161701, 2020.

[3] S. Long, J. Chen, A. Hu, H. P. Liu and D. C. Zheng, "Microaneurysms detection in color fundus images using machine learning based on directional local contrast”, BioMedical Engineering Online, vol. 19, no. 1, pp. 21, 2020.

[4] T. Song, L. Xin, C. Gao, T. Q. Zhang and Y. Huang, "Quaternionic extended local binary pattern with adaptive structural pyramid pooling for color image representation", Pattern Recognition, vol. 115, pp. 107891, 2021.

[5] Y. Kinoshita and H. Kiya, "Hue-correction scheme based on constant-hue plane for deep-learning-based color-image enhancement", IEEE Access, vol. 8, no. 9, pp. 9540-9550, 2020. 
[6] J. Anitha, S. Pandian and S. A. Agnes, "An efficient multilevel color image thresholding based on modified whale optimization algorithm", Expert Systems with Applications, vol. 12, no. 11, pp. 115003, 2021.

[7] N. Varish, A. K. Pal, R. Hassan, M. K. Hasan and I. Memon, "Image retrieval scheme using quantized bins of color image components and adaptive tetrolet transform”, IEEE Access, vol. 8, pp. 117639-117665, 2020.

[8] M. Arif and Y. Muhammad, "Modeling of the acute lymphoblastic leukemia detection based on the principal object characteristics of the color image", Procedia Computer Science, vol. 157, no. 11, pp. 87-98, 2019.

[9] K. Hosono, S. Ono and T. Miyata, "Weighted tensor nuclear norm minimization for color image restoration", IEEE Access, vol. 7, pp. 88768-88776, 2019.

[10] M. H. Khalid and M. D. Mohamed, "New set of multi-channel orthogonal moments for color image representation and recognition", Pattern Recognition, vol. 88, no. 10, pp. 153-173, 2019.

[11] G. Kaur, R. Agarwal and V. Patidar, "Color image encryption scheme based on fractional Hartley transform and chaotic substitution-permutation", The Visual Computer, vol. 23, no. 3, pp. 1-24, 2021.

[12] T. T. Nguyen, H. J. Wang, T. K. Dao, J. S. Pan and J. $\mathrm{Yu}$, "A scheme of color image multithreshold segmentation based on improved Moth-Flame algorithm", IEEE Access, vol. 8, no. 9, pp. 174142-174159, 2020.

[13] S. Hraoui, F. Gmira, M. F. Abbou, A. J. Oulidi and A. Jarjar, "A new cryptosystem of color image using a dynamic-chaos hill cipher algorithm", Procedia Computer Science, vol. 148, no. 9, pp. 399-408, 2019.

[14] H. R. Shakir, "A color-image encryption scheme using a 2D chaotic system and DNA coding", Advances in Multimedia, vol. 2019, no. 10, pp. 1-13, 2019.

[15]D. Mújica-Vargas, J. Kinani and J. Rubio, "Color-based Image segmentation by means of a robust intuitionistic fuzzy C-Means algorithm", International Journal of Fuzzy Systems, vol. 22, no. 3, pp. 901-916, 2020.

[16] L. K. Pavithra and S. T. Sree, "An improved seed point Selection-Based unsupervised color clustering for content-based image retrieval application", The Computer Journal, vol. 11, no. 3, pp. 3, 2019.

[17] I. A. Siradjuddin, R. P. Bahruddin and M. K. Sophan, "Combination of term weighting and integrated color intensity co-occurrence matrix for two-Level image retrieval on social media data", Procedia Computer Science, vol. 157, no. 5, pp. 329-336, 2019.

[18] H. T. Hu, L. Y. Hus and H. H. Chou, "An improved "SVD-based blind color image watermarking algorithm with mixed modulation incorporated", Information Sciences, vol. 519, no. 11, pp. 161-182, 2020.

[19] A. Molada, A. Marqués-Mateu, J. L. Lerma and S. Westland, "Dominant color extraction with K-Means for camera characterization in cultural heritage documentation", Remote Sensing, vol. 12, no. 3, pp. $520,2020$.
[20] N. Phadungcharoen, N. Pengwanput, A. Nakapan, U. Sutitaphan and T. Rojanarata, "Ion pair extraction coupled with digital image colorimetry as a rapid and green platform for pharmaceutical analysis: An example of chlorpromazine hydrochloride tablet assay", Talanta, vol. 219, no. 9, pp. 121271, 2020.

[21] S. Vetova, "Covid image classification using wavelet feature vectors and NN", Engineering World, vol. 3, pp. 38-42, 2021.

[22]Z. Guezoui, A. B. Goumeidane and N. Nacereddine, "Weld defect radiographic image segmentation with finite mixture model (FMM)", Engineering World, vol. 2, pp. 134-138, 2020.

\section{Creative Commons Attribution License 4.0 (Attribution 4.0 International, CC BY 4.0)}

This article is published under the terms of the Creative Commons Attribution License 4.0

https://creativecommons.org/licenses/by/4.0/deed.en_US 